\title{
ON THE MINIMUM ORDER OF EXTREMAL GRAPHS TO HAVE A PRESCRIBED GIRTH*
}

\author{
C. BALBUENA ${ }^{\dagger}$ AND P. GARCÍA-VÁZQUEZ
}

\begin{abstract}
We show that any $n$-vertex extremal graph $G$ without cycles of length at most $k$ has girth exactly $k+1$ if $k \geq 6$ and $n>\left(2(k-2)^{k-2}+k-5\right) /(k-3)$. This result provides an improvement of the asymptotical known result by Lazebnik and Wang [J. Graph Theory, 26 (1997), pp. 147-153] who proved thatthe girth is exactly $k+1$ if $k \geq 12$ and $n \geq 2^{a^{2}+a+1} k^{a}$, where $a=k-3-\lfloor(k-2) / 4\rfloor$. Moreover, we prove that the girth of $G$ is at most $k+2$ if $n>\left(2(t-2)^{k-2}+t-5\right) /(t-3)$, where $t=\lceil(k+1) / 2\rceil \geq 4$. In general, for $k \geq 5$ we show that the girth of $G$ is at most $2 k-4$ if $n \geq 2 k-2$.
\end{abstract}

Key words. extremal graphs, girth, forbidden cycles, cages

AMS subject classification. $05 \mathrm{C} 35$

DOI. $10.1137 / 060656747$

1. Introduction. Throughout this paper, only undirected simple graphs without loops or multiple edges are considered. Unless otherwise stated, we follow [2] for terminology and definitions.

Let $V(G)$ and $E(G)$ denote the set of vertices and the set of edges of a graph $G$, respectively. The order of $G$ is denoted by $|V(G)|=n$ and the size by $|E(G)|=e(G)$. The minimum length of a cycle contained in $G$ is the girth $g(G)$ of $G$. A cycle of minimum length is said to be a girdle and if $G$ does not contain a cycle, we set $g(G)=\infty$. By $C_{r}$ we will denote a cycle of length $r, r \geq 3$.

Let $\mathcal{F}$ be a family of graphs. The extremal number ex $(n, \mathcal{F})$ is the maximum number of edges in a graph of order $n$ that does not contain any graph of $\mathcal{F}$ as a subgraph. The graphs of order $n$ and size $\operatorname{ex}(n, \mathcal{F})$ not containing any $F \in \mathcal{F}$ as a subgraph are the extremal graphs and are denoted by $\operatorname{EX}(n, \mathcal{F})$. We refer to graphs from $\operatorname{EX}(n, \mathcal{F})$ as extremal $\mathcal{F}$-free graphs of order $n$, or just extremal.

By ex $\left(n ;\left\{C_{3}, C_{4}, \ldots, C_{k}\right\}\right)$ we denote the maximum number of edges in a graph of order $n$ and girth at least $k+1$, and by $\operatorname{EX}\left(n ;\left\{C_{3}, C_{4}, \ldots, C_{k}\right\}\right)$ we denote the set of all graphs of order $n$, girth at least $k+1$, and with $\operatorname{ex}\left(n ;\left\{C_{3}, C_{4}, \ldots, C_{k}\right\}\right)$ edges. Erdös and Sachs [3] showed that an $r$-regular graph of girth at least $k+1$ with the least possible number of vertices has girth equal to $k+1$. (A proof of this result can be found in Lovász [7, pp. 66, 384, 385, and the references therein].) These graphs are called $(r ; k+1)$-cages.

In this paper we consider a similar question asked by Garnick and Nieuwejaar in [5] on extremal graphs with a relatively large girth. Is there a constant $c$ such that for all $k \geq 5$ and all $n \geq c k$, the girth of any extremal graph with girth $\geq k+1$ is $k+1$ ? They give an affirmative answer for $k=4$. Lazebnik and Wang [6] showed that the

*Received by the editors April 7, 2006; accepted for publication (in revised form) October 11, 2006; published electronically April 6, 2007. This research was supported by the Ministry of Education and Science, Spain, and the European Regional Development Fund (ERDF) under project MTM200508990-C02-02.

http://www.siam.org/journals/sidma/21-1/65674.html

${ }^{\dagger}$ Departament de Matemàtica Aplicada III, Universitat Politècnica de Catalunya, Barcelona, Spain (m.camino.balbuena@upc.edu).

${ }_{\ddagger}$ Departamento de Matemática Aplicada I, Universidad de Sevilla, Sevilla, Spain (pgvazquez@ us.es). 
answer is negative for $c=2$ and affirmative if $k=5$ or if $n$ is large in comparison with $k$. More precisely they proved the following result.

TheOrem A. Let $k \geq 12, \quad a=k-3-\lfloor(k-2) / 4\rfloor, \quad n \geq 2^{a^{2}+a+1} k^{a}$, and $G \in \operatorname{EX}\left(n ;\left\{C_{3}, C_{4}, \ldots, C_{k}\right\}\right)$. Then the girth $g(G)=k+1$.

In order to prove Theorem A, Lazebnik and Wang used the following result, which they also stated in [6].

Theorem B. Let $k \geq 3, G \in E X\left(n ;\left\{C_{3}, C_{4}, \ldots, C_{k}\right\}\right)$, and the maximum degree be $\Delta(G) \geq k$. Then $g(G)=k+1$.

Our main contribution to this problem is to provide an improvement of Theorem A. More precisely we prove that the girth of $G \in E X\left(n ;\left\{C_{3}, C_{4}, \ldots, C_{k}\right\}\right)$ is $k+1$ if either $k=3$ and $n \geq 5$; or $k=4$ and $n \geq 9$; or $k=5$ and $n \geq 8$; or $k=6$ and $n \geq 171$; or $k \geq 7$ and

$$
n \geq \frac{2(k-2)^{k-2}+k-5}{k-3}+1 .
$$

This contribution contains the known results for $k=3,4,5$; see $[4,5,6]$. Furthermore, it gives an answer to the problem for $k=6$ posed by Lazebnik and Wang [6], who asked to prove the girth of an extremal $\left\{C_{3}, C_{4}, C_{5}, C_{6}\right\}$-free graph is 7 .

Moreover, we show that the girth of $G \in \operatorname{EX}\left(n ;\left\{C_{3}, C_{4}, \ldots, C_{k}\right\}\right)$ is at most $2 k-4$ provided that $k \geq 5$ and $n \geq 2 k-2$. This clearly implies that for $k=6$ the girth of an extremal graph is at most 8 for $10 \leq n \leq 170$.

Let $t=\lceil(k+1) / 2\rceil$. We also prove that the girth of $G \in \operatorname{EX}\left(n ;\left\{C_{3}, C_{4}, \ldots, C_{k}\right\}\right)$ is at most $k+2$ if $k \geq 7$ and

$$
n \geq \frac{2(t-2)^{k-2}+t-5}{t-3}+1 .
$$

From this result it follows for $k=7$ that if $n \geq 64$, then $g(G) \leq 9$.

2. Main results. The set of neighbors of $u \in V(G)$ is denoted by $N_{G}(u)$. The number of neighbors of $u$ is the degree $d_{G}(u)$ of $u$ in $G$, or briefly $d(u)$ when it is clear which graph is meant. The distance $d_{G}(x, y)$ in $G$ of two vertices $x, y$ is the length of a shortest $x-y$ path in $G$. The greatest distance between any two vertices in $G$ is the diameter $D(G)$ of $G$. Diameter and girth are related by $g(G) \leq 2 D(G)+1$. Let $e=x y$ be an edge of $G$. As usual we will denote by $G /\{e\}=G / e$ the graph obtained from $G$ by contracting the edge $e$ into a new vertex $v_{e}$, which becomes adjacent to all the former neighbors of $x$ and $y$. Taking into account that we dealt with simple graphs of girth at least 4 the resultant graph by any edge contraction remains simple.

Throughout the paper $k \geq 3$ is an integer. We begin by proving a technical and useful lemma.

Lemma 2.1. Let $G \in E X\left(n ;\left\{C_{3}, \ldots, C_{k}\right\}\right)$ have two distinct edges $e_{1}$ and $e_{2}$ such that every cycle of $G$ containing both of them has a length of at least $k+3$. Then the girth is $g(G)=k+1$ if the diameter is $D\left(G /\left\{e_{1}, e_{2}\right\}\right) \geq k-2$.

Proof. Let $G \in E X\left(n ;\left\{C_{3}, \ldots, C_{k}\right\}\right)$ satisfy the hypothesis of the lemma and suppose that the girth is $g(G) \geq k+2$. The graph $G^{\prime}=G /\left\{e_{1}, e_{2}\right\}$ has $g\left(G^{\prime}\right) \geq k+1$ because by hypothesis any cycle passing through both edges $e_{1}$ and $e_{2}$ has a length of at least $k+3$. Let $u^{\prime}, v^{\prime}$ be two vertices of $G^{\prime}$ such that $d_{G^{\prime}}\left(u^{\prime}, v^{\prime}\right)=D\left(G^{\prime}\right)$; then by hypothesis $d_{G^{\prime}}\left(u^{\prime}, v^{\prime}\right)=D\left(G^{\prime}\right) \geq k-2$. Let us consider the graph $G^{*}$ obtained from $G^{\prime}$ by adding two new vertices $x_{1}, x_{2}$ and the three edges $u^{\prime} x_{1}, x_{1} x_{2}$, and $x_{2} v^{\prime}$. We have $g\left(G^{*}\right)=\min \left\{g\left(G^{\prime}\right), D\left(G^{\prime}\right)+3\right\} \geq k+1,\left|V\left(G^{*}\right)\right|=\left|V\left(G^{\prime}\right)\right|$ 
$+2=n$, and $e\left(G^{*}\right)=e(G)+1$, which contradict the maximality of $G$. Therefore $g(G)=k+1$.

As a first consequence of the above lemma, we obtain in the next theorem an upper bound for the girth of any extremal graph which contains the known result $g=k+1$ for $k=5$; see [6].

Theorem 2.2. Let $G \in E X\left(n ;\left\{C_{3}, \ldots, C_{k}\right\}\right)$ be for $k \geq 5$ and $n \geq 2 k-2$. Then $G$ has a girth of $g(G) \leq 2 k-4$.

Proof. Let $G \in E X\left(n ;\left\{C_{3}, \ldots, C_{k}\right\}\right)$ satisfy the hypothesis of the theorem, and assume the girth of $G$ is $g \geq 2 k-2$. Let $C: u_{0} u_{1} \cdots u_{g-1} u_{0}$ be a girdle in $G$, and notice that $g \geq k+3$ because $k \geq 5$. The graph $G^{\prime}=G /\left\{u_{0} u_{1}, u_{1} u_{2}\right\}$ clearly has girth $g\left(G^{\prime}\right) \geq 2 k-4$; hence the diameter is $D\left(G^{\prime}\right) \geq\left\lfloor g\left(G^{\prime}\right) / 2\right\rfloor \geq\lfloor(2 k-4) / 2\rfloor=k-2$. By Lemma 2.1 we have $g=g(G)=k+1$, yielding $2 k-2 \leq k+1$, which is a contradiction because $k \geq 5$. Therefore the girth of $G$ is $g \leq 2 k-3$. Assume the girth of $G$ is exactly $g=2 k-3$. As $n \geq 2 k-2$ the graph $G$ must contain a vertex $y$ not belonging to $C$. Without loss of generality, suppose that $u_{0} y$ is an edge of $G$. Notice that $u_{k-2}$ and $u_{k-1}$, both belonging to $C$, satisfy that $d_{C}\left(u_{0}, u_{k-2}\right)=d_{C}\left(u_{0}, u_{k-1}\right)=k-2$. Then both $u_{0}-u_{k-2}$ and $u_{0}-u_{k-1}$ paths contained in $C$ must be the unique shortest $u_{0}-u_{k-2}$ and $u_{0}-u_{k-1}$ paths in $G$, because $k-2=(g-1) / 2$. This implies that $d_{G}\left(y, u_{k-2}\right) \geq k-2$ and $d_{G}\left(y, u_{k-1}\right) \geq k-2$ so that every cycle, if any, containing both edges $u_{0} y$ and $u_{k-2} u_{k-1}$ must have a length of at least $g+1=2 k-2$, which is at least $k+3$ because $k \geq 5$. Now let $G^{\prime \prime}=G /\left\{u_{0} y, u_{k-2} u_{k-1}\right\}$. Clearly, $D\left(G^{\prime \prime}\right) \geq$ $d_{G^{\prime \prime}}\left(u_{1}, u_{k}\right)=d_{G}\left(u_{1}, u_{k}\right)=k-2$. By Lemma 2.1 we obtain $g(G)=g=k+1$, i.e., $2 k-3 \leq k+1$, which is impossible for $k \geq 5$. Hence the girth of $G$ is at most $2 k-4$ and the theorem is valid.

Next, we obtain the following result which is an improvement of Theorem A and also contains the known results for $k=3,4,5$; see $[4,5,6]$.

Theorem 2.3. Let $G \in E X\left(n ;\left\{C_{3}, \ldots, C_{k}\right\}\right)$. Then $g(G)=k+1$ if either $k=3$ and $n \geq 5$; or $k=4$ and $n \geq 9$; or $k=5$ and $n \geq 8$; or $k=6$ and $n \geq 171$; or $k \geq 7$ and

$$
n \geq \frac{2(k-2)^{k-2}+k-5}{k-3}+1 .
$$

Proof. From Theorem 2.2 it follows that any graph $G \in E X\left(n ;\left\{C_{3}, C_{4}, C_{5}\right\}\right)$ for $n \geq 8$ has girth of 6 . Therefore we can assume $k=3,4$ or $k \geq 6$. Let $G \in$ $E X\left(n ;\left\{C_{3}, \ldots, C_{k}\right\}\right)$ and suppose that its girth is $g(G) \geq k+2$. Then, by Theorem B we have $\Delta \leq k-1$, where $\Delta$ denotes the maximum degree of $G$. Let $D$ be the diameter of $G$ and let us take two vertices $x, y$ at distance $d_{G}(x, y)=D$. Then $D \leq k-1$ because otherwise by adding the edge $x y$ to $G$ we would obtain a graph $G^{\prime}$ of order $n$ having girth $g\left(G^{\prime}\right) \geq k+1$ and more edges than $G$, which contradicts the maximality of $G$. Let us consider the two cases $D=k-1$ and $D \leq k-2$ separately.

Case 1. $D=k-1$. Define the set $N_{G}^{k-1}(x)=\left\{y \in V(G): d_{G}(x, y)=k-1\right\}$. Clearly, $\left|N_{G}^{k-1}(x)\right| \geq 1$, because $y \in N_{G}^{k-1}(x)$. Let us see that $\left|N_{G}^{k-1}(x)\right|=1$.

Let $W=\left\{w \in V(G): d_{G}(x, w)+d_{G}(w, y)=k-1\right\}$ and suppose that there exists a vertex $u \in V(G) \backslash W$. Then $d_{G}(x, u)+d_{G}(u, y) \geq k$ or, in other words, all the possible paths passing through $u$ that connect $x$ with $y$ have a length of at least $k$. Take any vertex $v \in N_{G}(u)$ and consider the graph $G^{\prime}$ resulting by contracting the edge $u v$ in $G$. The girth of this new graph is $g\left(G^{\prime}\right) \geq k+1$ and the diameter $D\left(G^{\prime}\right)=D=k-1$. So let $x^{\prime}, y^{\prime} \in V\left(G^{\prime}\right)$ be such that $d_{G^{\prime}}\left(x^{\prime}, y^{\prime}\right)=k-1$, and denote by $G^{*}$ the graph obtained from $G^{\prime}$ by adding a new vertex $x^{*}$ and the edges 
$x^{\prime} x^{*}$ and $x^{*} y^{\prime}$. Clearly, $\left|V\left(G^{*}\right)\right|=\left|V\left(G^{\prime}\right)\right|+1=n$, and girth $g\left(G^{*}\right)=k+1$, but $e\left(G^{*}\right)=e\left(G^{\prime}\right)+2=e(G)+1$, which contradicts the maximality of $G$. Hence, $V(G)=W$, which readily implies that $y$ is the only vertex at distance $D=k-1$ from $x$ and the number of vertices at distance $D-1=k-2$ from $x$ is at most $\Delta$, since these vertices must be neighbors of $y$.

Therefore, if $k=3$, then $n \leq 1+\Delta+1 \leq 1+k=4$, contradicting the hypothesis for this case. If $k=4$, then $n \leq 1+\Delta+\Delta+1 \leq 2 k=8$, contradicting again the hypothesis for this case. So assume that $k \geq 6$. As for $1 \leq i \leq D-2=k-3$, the maximum number of vertices at distance $i$ from $x$ is $\Delta(\Delta-1)^{i-1}$, we obtain

$$
\begin{aligned}
n & \leq 1+\Delta \sum_{i=0}^{k-4}(\Delta-1)^{i}+\Delta+1 \leq 1+(k-1) \sum_{i=0}^{k-4}(k-2)^{i}+k \\
& =\frac{(k-1)(k-2)^{k-3}-2}{k-3}+k \\
& <\frac{(k-1)(k-2)^{k-3}-2}{k-3}+(k-2)^{k-3}=\frac{2(k-2)^{k-2}-2}{k-3} .
\end{aligned}
$$

This contradicts the hypothesis of the theorem, so $g(G)=k+1$ in the case $D=k-1$.

Case 2. $D \leq k-2$. Notice that $k=3,4$ are impossible for this case because $D \geq\lfloor g / 2\rfloor \geq\lfloor(k+2) / 2\rfloor$. So we have $k \geq 6$.

Let $x^{*}$ be a vertex of $G$ with degree $d_{G}\left(x^{*}\right)=\delta$, where $\delta$ is the minimum degree of $G$, and let us denote by $\epsilon\left(x^{*}\right)=\max \left\{d_{G}\left(x^{*}, y\right): y \in V(G)\right\}$ the eccentricity of $x^{*}$. As the diameter is the maximum of the eccentricities we have $\epsilon\left(x^{*}\right) \leq D \leq k-2$. Suppose first that $\epsilon\left(x^{*}\right) \leq k-3$. As for $1 \leq i \leq k-3$, the maximum number of vertices at distance $i$ from $x^{*}$ is $\delta(\Delta-1)^{i-1}$, it is immediate that

$$
n \leq 1+\delta \sum_{i=0}^{k-4}(\Delta-1)^{i} \leq 1+(k-1) \sum_{i=0}^{k-4}(k-2)^{i} \leq \frac{(k-1)(k-2)^{k-3}-2}{k-3},
$$

which is a contradiction. Therefore $\epsilon\left(x^{*}\right)=k-2$, which means $D=k-2$. Let us consider the set $N_{G}^{k-2}\left(x^{*}\right)=\left\{y \in V(G): d_{G}\left(x^{*}, y\right)=k-2\right\}$. Let us prove the following claim.

Claim. Given any vertex $y \in N_{G}^{k-2}\left(x^{*}\right)$, every neighbor of vertex $y$ is at a distance of $k-3$ from $x^{*}$.

Otherwise suppose that there exists a vertex $y_{1} \in N_{G}^{k-2}\left(x^{*}\right) \cap N_{G}(y)$. Let us denote by $x^{*}=x_{0} x_{1} x_{2} \cdots x_{k-2}=y$ any shortest $x^{*}-y$ path. Clearly, every cycle containing both edges $x^{*} x_{1}$ and $y y_{1}$, if any, has a length of at least $k+3$ because $k \geq 6$. Then we consider the new graph $G^{\prime}$ obtained from $G$ by contracting the edges $x^{*} x_{1}$ and $y y_{1}$. If the diameter of $G^{\prime}$ is $D\left(G^{\prime}\right)=k-2$, then by Lemma 2.1 we would have $g(G)=k+1$, which is a contradiction with our assumption $g(G) \geq k+2$. Therefore $D\left(G^{\prime}\right)=k-3$, which implies that for all $z \in N\left(x^{*}\right), d_{G}\left(z, y^{\prime}\right)=k-3$ for all $y^{\prime} \in N_{G}^{k-2}\left(x^{*}\right)$. Consequently, the edge $y y_{1}$ and any vertex $z \in N_{G}\left(x^{*}\right)$ lies on a cycle in $G$ of length at most $2 k-5$, which is impossible for $k=6$ because $g \geq k+2$. Hence every neighbor of vertex $y$ is at a distance of $k-3$ from $x^{*}$ when $k=6$ and the claim is true for this case.

Furthermore, for $k \geq 7$ we have $d_{G^{\prime}}\left(v_{x^{*} x_{1}}, v_{y y_{1}}\right)=k-3$, where $v_{x^{*} x_{1}}$ and $v_{y y_{1}}$ denote the newly arising vertices by the contraction of the edges $x^{*} x_{1}$ and $y y_{1}$. Besides, $d_{G^{\prime}}\left(v_{x^{*} x_{1}}\right)=d_{G}\left(x^{*}\right)+d_{G}\left(x_{1}\right)-2 \leq \delta+\Delta-2 \leq 2(\Delta-1)$ and $d_{G^{\prime}}\left(v_{y y_{1}}\right)=$ 
$d_{G}(y)+d_{G}\left(y_{1}\right) \leq 2(\Delta-1)$. Therefore,

$$
V\left(G^{\prime}\right)=\left\{v_{x^{*} x_{1}}\right\} \cup \bigcup_{i=1}^{k-3} N_{G^{\prime}}^{i}\left(v_{x^{*} x_{1}}\right),
$$

where $N_{G^{\prime}}^{i}\left(v_{x^{*} x_{1}}\right)$ denotes the set of vertices of $G^{\prime}$ at a distance of $i$ from vertex $v_{x^{*} x_{1}}$. Thus $\left|N_{G^{\prime}}^{i}\left(v_{x^{*} x_{1}}\right)\right| \leq 2(\Delta-1)(\Delta-1)^{i-1}=2(\Delta-1)^{i}$, for $i=1, \ldots, k-3$, and we get

$$
\begin{aligned}
n & =2+\left|V\left(G^{\prime}\right)\right| \leq 3+2 \sum_{i=1}^{k-3}(\Delta-1)^{i} \\
& \leq 3+2 \sum_{i=1}^{k-3}(k-2)^{i} \\
& =3+\frac{2(k-2)^{k-2}-2(k-2)}{k-3}=\frac{2(k-2)^{k-2}+k-5}{k-3},
\end{aligned}
$$

contradicting the hypothesis of the theorem. Thus, every vertex $y \in N_{G}^{k-2}\left(x^{*}\right)$ has all its neighbors at distance $k-3$ from $x^{*}$ and the claim holds.

Hence, $\left|N_{G}^{i}\left(x^{*}\right)\right| \leq \delta(\Delta-1)^{i-1}$, for $i=1, \ldots, k-3$, and $\left|N_{G}^{k-2}\left(x^{*}\right)\right| \leq(\Delta-1)^{k-3}$. Then, for $k \geq 6$ we have

$$
\begin{aligned}
n & \leq 1+\delta \sum_{i=0}^{k-4}(\Delta-1)^{i}+(\Delta-1)^{k-3} \\
& \leq 1+\delta \sum_{i=0}^{k-4}(k-2)^{i}+(k-2)^{k-3} \\
& \leq \frac{(k-1)(k-2)^{k-3}-2}{k-3}+(k-2)^{k-3}=\frac{2(k-2)^{k-2}-2}{k-3} .
\end{aligned}
$$

This contradicts the hypothesis of the theorem, so we conclude that $g(G)=$ $k+1$.

Next, the goal is to provide a lower bound on $n$ in order to guarantee that the girth is at most $k+2$ for $k \geq 7$. To do that first we state that an extremal $\left\{C_{3}, \ldots, C_{k}\right\}$-free graph with maximum degree $\Delta \geq\lceil(k+1) / 2\rceil$ has necessarily a girth of at most $k+2$.

TheOREM 2.4. Let $k \geq 7$ be an integer. Let $G$ be a graph belonging to the family $E X\left(n ;\left\{C_{3}, \ldots, C_{k}\right\}\right)$ with a minimum degree of at least 2 and maximum degree $\Delta$. Then $g(G) \leq k+2$ if $\Delta \geq\lceil(k+1) / 2\rceil$.

Proof. Let $G \in E X\left(n ;\left\{C_{3}, \ldots, C_{k}\right\}\right)$ satisfy the hypothesis of the theorem, and assume $g(G) \geq k+3$. Let $x$ be a vertex of maximum degree $\Delta$ and let $y_{1}, y_{2}, \ldots, y_{\Delta}$ be all the neighbors of $x$. Since $d_{G}\left(y_{i}\right) \geq 2$, for each $i=1, \ldots, \Delta$, there exists $x_{i} \in V(G)-x$ adjacent to $y_{i}$. Notice also that $x_{i} \neq x_{j}$ for all $i \neq j$, since $g(G)>4$. Taking into account that $g(G) \geq k+3$, we deduce that $d_{G-x}\left(x_{i}, x_{j}\right) \geq g(G)-4 \geq$ $k-1, d_{G-x}\left(y_{i}, y_{j}\right) \geq g(G)-2 \geq k+1$, and $d_{G-x}\left(x_{i}, y_{j}\right) \geq g(G)-3 \geq k$ for all $i, j=1, \ldots, \Delta$ with $i \neq j$. Let $G^{*}$ be the graph obtained from $G$ by first deleting the $\Delta-1$ edges $x y_{2}, \ldots, x y_{\Delta}$ and second adding the new $\Delta$ edges $y_{1} x_{2}, \ldots, y_{\Delta-1} x_{\Delta}, y_{\Delta} x_{1}$. Then $G^{*}$ has order $n$ and size $e\left(G^{*}\right)=e(G)+1$. Since $G$ is extremal, $G^{*}$ must contain a cycle of length at most $k$. Let us denote by $C^{*}$ a shortest cycle in $G^{*}$ (notice that 
$x \notin V\left(C^{*}\right)$, since $x$ has degree 1 in $\left.G^{*}\right)$. We denote by $C$ the cycle $x_{1} y_{1} x_{2} y_{2} \cdots x_{\Delta} y_{\Delta} x_{1}$ which has length $2 \Delta \geq k+1$. Observe that $C$ is an induced cycle of $G^{*}$, since $x_{i}$ is nonadjacent to $y_{j}$ in $G$, for any $i \neq j$ and the only newly introduced edges are $y_{i} x_{i+1}$ for $i=1, \ldots, \Delta-1$ and $y_{\Delta} x_{1}$. Moreover, $C^{*} \neq C$, since $g(C) \geq k+1$ and $g\left(C^{*}\right) \leq k$. So, we may express $C^{*}=P_{1} \cup P_{2}$, where $P_{1}$ is the longest path whose edges belong to the set $E\left(C^{*}\right) \backslash E(C) \subseteq E(G-x)$, and $P_{2}$ is the rest of $C^{*}$. Notice that the endvertices of $P_{1}$ must belong to $\left\{x_{1}, \ldots, x_{\Delta}\right\} \cup\left\{y_{1}, \ldots, y_{\Delta}\right\}$ by the construction of $P_{1}$. Observe also that $P_{2}$ contains at least one edge of $E(C)$, because otherwise the cycle $C^{*}$ would be contained in $G$ against the assumption $g(G) \geq k+3$. If the endvertices of $P_{1}$ are $x_{i}$ and $x_{j}$ for certain $i, j \in\{1,2 \ldots, \Delta\}$, then the edge $y_{i-1} x_{i}$ or $x_{i} y_{i}$ and the edge $y_{j-1} x_{j}$ or $x_{j} y_{j}$ must be contained in $P_{2}$ and then $e\left(P_{2}\right) \geq 2$. This implies that $\left|V\left(C^{*}\right)\right|=e\left(C^{*}\right)=e\left(P_{1}\right)+e\left(P_{2}\right) \geq d_{G-x}\left(x_{i}, x_{j}\right)+2 \geq k-1+2=k+1$; a contradiction. If the endvertices of $P_{1}$ are $x_{i}$ and $y_{i}$, for some $i \in\{1, \ldots, \Delta\}$, then $e\left(P_{1}\right) \geq d_{G-x-\left\{x_{i} y_{i}\right\}}\left(x_{i}, y_{i}\right) \geq g(G)-1 \geq k+2$ and hence $\left|V\left(C^{*}\right)\right|=e\left(C^{*}\right)=$ $e\left(P_{1}\right)+e\left(P_{2}\right) \geq k+3$, again a contradiction. Otherwise,

$$
e\left(P_{1}\right) \geq \min \left\{d_{G-x}\left(y_{i}, y_{j}\right), d_{G-x}\left(x_{i}, y_{j}\right): i, j=1, \ldots, \Delta \text { and } i \neq j\right\} \geq k,
$$

which implies $\left|V\left(C^{*}\right)\right|=e\left(C^{*}\right)=e\left(P_{1}\right)+e\left(P_{2}\right) \geq k+1>k$, arriving at a contradiction. Hence, $g(G) \leq k+2$.

From Theorem 2.4 we derive the following sufficient condition in terms of the order for an extremal $\left\{C_{3}, \ldots, C_{k}\right\}$-free graph to have girth at most $k+2$.

TheOREM 2.5. Let $G \in E X\left(n ;\left\{C_{3}, \ldots, C_{k}\right\}\right)$ be of a minimum degree of at least 2. Then the girth is $g(G) \leq k+2$ if $k \geq 7$ and

$$
n \geq \frac{2(t-2)^{k-2}+t-5}{t-3}+1
$$

where $t=\lceil(k+1) / 2\rceil$.

Proof. If $\Delta \geq\lceil(k+1) / 2\rceil$, then $g(G) \leq k+2$ for $k \geq 7$ because of Theorem 2.4 and the theorem holds. Hence assume $\Delta \leq\lceil(k+1) / 2\rceil-1$ and $g(G) \geq k+3$. Let $t=\lceil(k+1) / 2\rceil$. As in the proof of Theorem 2.3 we consider two cases $D=k-1$ and $D \leq k-2$ separately and repeat this proof but taking into account that now $\Delta \leq t-1$ instead of $\Delta \leq k-1$. In this way we arrive at a contradiction, which implies $g(G) \leq k+2$, and the theorem holds.

As an immediate consequence of Theorems 2.3 and 2.5, the following information about the girth of any extremal $\left\{C_{3}, \ldots, C_{7}\right\}$-free graph is provided.

Corollary 2.6. Let $G$ be a graph belonging to the family $E X\left(n ;\left\{C_{3}, \ldots, C_{7}\right\}\right)$. Then the girth $g(G)=8$ if $n \geq 783$, and the girth is $g(G) \leq 9$ if $n \geq 64$.

3. Conclusions. Theorem 2.3 can be compared with Theorem A. Both results give a sufficient condition on the order of an extremal graph to contain a cycle of minimum length $k+1$. Recall that $a=k-3-\lfloor(k-2) / 4\rfloor$; then for $k \geq 12$ we have $2^{a}>(k-2)^{2}$. Hence $2^{a^{2}+a+1}>2(k-2)^{2 a+2} \geq 2(k-2)^{(3 k-6) / 2}$, and thus $n \geq 2^{a^{2}+a+1} k^{a}>2(k-2)^{(3 k-6) / 2} k^{a}$ (which is much larger than the requirement obtained in Theorem 2.3), $n>\left(2(k-2)^{k-2}+k-5\right) /(k-3)$.

Moreover, Theorems 2.2 and 2.3 provide information on the girth of any extremal $\left\{C_{3}, C_{4}, C_{5}, C_{6}\right\}$-free graph $G$. The girth is $g(G)=7$ if $n \geq 171$, and the girth is $g(G) \leq 8$ if $n \geq 10$. It is known for $r=3,4,5$ that each $(r ; 8)$-cage is the incidence graph of a projective geometry called generalized quadrangle; see the survey by Wong [8]. The order of each of these graphs is $30,80,170$, respectively. As a referee suggests, 
it appears that a result of Alon, Hoory, and Linial [1] can be used to show these cages do belong to $\operatorname{EX}\left(n ;\left\{C_{3}, C_{4}, C_{5}, C_{6}, C_{7}\right\}\right)$. The question is if these cages are also $\left\{C_{3}, C_{4}, C_{5}, C_{6}\right\}$-free extremal. We would like to suggest the following open problems.

Problem 1. Prove or disprove that each $(r ; 8)$-cage for $r=3,4,5$ is a graph belonging to $E X\left(n ;\left\{C_{3}, C_{4}, C_{5}, C_{6}\right\}\right)$, for $n=30,80,170$.

Problem 2. Is it possible to improve the lower bound on $n$ in Theorem 2.3 for $k \geq 7$ ?

Acknowledgments. We would like to express our thanks to the referees for their helpful comments and suggestions.

\section{REFERENCES}

[1] N. Alon, S. Hoory, And N. Linial, The Moore bound for irregular graphs, Graphs Combin., 18 (2002), pp. 53-57.

[2] R. Diestel, Graph Theory, 3rd ed., Springer-Verlag, Berlin, 2005.

[3] P. Erdös And H. SAChs, Reguläre Graphen gegebener Taillenweite mit minimaler Knotenzahl, Wiss. Z. Martin-Luther-Univ. Halle-Wittenberg Math.-Natur. Reihe, 12 (1963), pp. 251257.

[4] D. K. Garnick, Y. H. H. Kwong, and F. Lazebnik, Extremal graphs without three-cycles or four-cycles, J. Graph Theory, 17 (1993), pp. 633-645.

[5] D. K. Garnick and N. A. NieunejaAR, Nonisomorphic extremal graphs without three-cycles or four-cycles, J. Combin. Math. Combin. Comput., 12 (1993), pp. 33-56.

[6] F. LAZEBNiK AND P. WANG, On the structure of extremal graphs of high girth, J. Graph Theory, 26 (1997), pp. 147-153.

[7] L. Lovász, Combinatorial Problems and Exercises, North-Holland, Amsterdam, 1979.

[8] P. K. Wong, Cages-A survey, J. Graph Theory, 6 (1982), pp. 1-22. 